\title{
Frecuencia de lesiones dermatológicas en pacientes adultos de cuidado intensivo
}

\author{
Frequency of dermatological lesions in adult \\ intensive care patients
}

\author{
Édgar Olmos Olmos, Mario Gómez-Duque, Mónica Rodríguez-Chávez \\ - Bogotá. D.C. (Colombia)
}

\section{Resumen}

Objetivo: describir la frecuencia de las lesiones dermatológicas en pacientes de la Unidad de Cuidados Intensivos (UCI) de adultos del Hospital de San José y del Hospital Infantil Universitario de San José entre enero y abril de 2012.

Pacientes y métodos: se realizó un estudio descriptivo de corte transversal. Se incluyeron pacientes mayores de 18 años con estancia mínima de 72 horas en UCI, ingresados por patología dermatológica o valorados por dermatología por aparición de lesiones en piel durante su estancia.

Resultados: se evaluaron 595 pacientes, encontrando lesiones dermatológicas en $30 \%$ (182 pacientes). La edad promedio fue de 63.5 años \pm 19.1 sin diferencias de género. La admisión a la UCI se debió principalmente a motivos no quirúrgicos con un porcentaje de $57.3 \%$ (105 pacientes). Las lesiones cutáneas más frecuentes fueron las lesiones terciarias observada en 91.8\% (168 pacientes), seguida por lesiones secundarias, con predominio infeccioso $6 \%$ (11 pacientes) y las últimas fueron las primarias con sólo $1.6 \%$ (3 pacientes).

Conclusiones: las lesiones dermatológicas en los pacientes de UCI son más frecuentes de lo esperado y en algunos casos pueden ser la causa directa del ingreso a la UCI poniendo en riesgo la vida de los pacientes, por lo tanto, consideramos importante un examen exhaustivo de piel en estos pacientes y el trabajo interdisciplinario con dermatología que permita disminuir el riesgo de mortalidad a causa de complicaciones de origen dermatológico. (Acta Med Colomb 2019; 44: 25-30).

Palabras clave: cuidados intensivos, dermatología, lesiones, frecuencia, corte transversal, enfermedad dermatológica.

\section{Abstract}

Objective: to describe the frequency of dermatological lesions in patients of the Intensive Care Unit (ICU) of adults of the Hospital de San José and the Hospital Infantil Universitario de San José between January and April 2012.

Patients and Methods: a descriptive cross-sectional study was carried out. Patients older than 18 years with a minimum stay of 72 hours in the ICU admitted for dermatological pathology or assessed by dermatology due to the appearance of skin lesions during their stay, were included.

Results: 595 patients were evaluated, finding dermatological lesions in 30\% (182 patients). The average age was 63.5 years \pm 19.1 without gender differences. Admission to the ICU was mainly due to non-surgical reasons with a percentage of $57.3 \%$ (105 patients). The most frequent skin lesions were the tertiary lesions observed in $91.8 \%$ (168 patients), followed by secondary lesions, with an infectious predominance of $6 \%$ (11 patients) and the last ones were the primary ones with only $1.6 \%$ (3 patients).

Conclusions: dermatological lesions in ICU patients are more frequent than expected and in some cases may be the direct cause of admission to the ICU putting patients' lives at risk; therefore, we consider important a thorough skin examination in these patients and interdisciplinary work with dermatology to reduce the risk of mortality due to dermatological complications. (Acta Med Colomb 2019; 44: 25-30).

Keywords: intensive care, dermatology, injuries, frequency, cross sectional study, dermatological disease.
Dr. Édgar Olmos-Olmos: Especialista en Dermatología, Universidad Javeriana. Jefe del Servicio de Dermatología, Hospital de San José Docente Fundación Universitaria de Ciencias de la Salud, Hospital de San José; Dr. Mario Gómez-Duque: Especialista en Anestesiología, Colegio Mayor de Nuestra Señora del Rosario. Especialista en Cuidado Intensivo, Fundación Universitaria de Ciencias de la Salud. Jefe de la Unidad de Cuidados Intensivos, Hospital de San José. Docente Fundación Universitaria de Ciencias de la Salud, Hospital de San José. Bogotá; Dra. Mónica Rodríguez-Chávez. Residente de Dermatología, Fundación Universitaria de Ciencias de la Salud, Hospital de San José. Bogotá. D.C. (Colombia).

Correspondencia: Dra. Mónica RodríguezChávez, Bogotá. D.C. (Colombia).

E-mail: monirodri14@gmail.com

Recibido: 17/X/2017 Aceptado: 16/I/2019 


\section{Introducción}

La insuficiencia cutánea aguda se define como una alteración de la función de la barrera cutánea que conduce a la pérdida de fluido, incapacidad para mantener la temperatura corporal, deterioro hemodinámico, y un mayor riesgo de infección que con frecuencia se acompaña de respuesta inflamatoria sistémica y puede ser causa de disfunción multiorgánica (1-3).

Su incidencia en Colombia, no es bien conocida y puede ser causada por lesiones primarias que tienen su origen en la piel como el pénfigo vulgar, síndrome de Steven-Johnson (SSJ), necrolisis epidérmica tóxica (NET) y la piel escaldada estafilocócica (4-6). También se puede originar por lesiones secundarias a enfermedades sistémicas como en el caso de la endocarditis bacteriana o la diabetes mellitus y por último a lesiones terciarias producidas por procedimientos médicos como catéteres, monitorización e intubación o mal manejo de la piel (7-10), todas requieren manejo intrahospitalario $\mathrm{y}$ algunas pueden llegar a requerir manejo en UCI especialmente si presentan complicación.

Existen otros tipos de lesiones dermatológicas, que se generan de manera secundarias al manejo médico o inadecuado cuidado en las UCI, producidas por el uso de diferentes medicamentos o dispositivos con inadecuada higiene $o$ sobreinfección $(4,7,11,12)$

Es importante la detección y tratamiento temprano de la falla cutánea debido al potencial de riesgo para la vida, para ello, nos podemos apoyar en los signos de compromiso sistémico que pueden acompañar a la patología dermatológica como indicador de enfermedad (6).

En el caso de los pacientes críticos, se conoce que la piel es más vulnerable al daño externo y su reparación está alterada, incrementando el riesgo de complicaciones (13, 14). Por lo tanto, el examen dermatológico se debe realizar de manera rutinaria para la detección temprana de lesiones en piel que se pueden complicar y generar falla cutánea aguda y muerte $(4,6,13)$.

Por otra parte, se ha evidenciado que las lesiones dermatológicas en UCI, tienden a ser subestimadas (15) y poco registradas en la mayoría de los casos, lo que evita un tratamiento temprano y adecuado pudiendo afectar de manera directa el pronóstico (16-18). Además debido a la sedación e intubación orotraqueal, los pacientes no pueden expresar sintomatología, ni aclarar antecedentes que orienten al clínico a un posible diagnóstico como por ejemplo el uso de: antibióticos (betalactámicos, macrólidos y lincosamidas especialmente), antihipertensivos (principalmente bloqueadores de canales de calcio), antimaláricos y anticonvulsivantes, que son los principales medicamentos causantes de lesiones dermatológicas (19).

Todos éstos factores dificultan el diagnóstico principalmente de las lesiones por hipersensibilidad a medicamentos y la exclusión de diagnósticos diferenciales $(20,21)$. Por todos estos motivos, se resalta la importancia del acompañamiento por parte del especialista en dermatología que apoye al servicio tratante en el diagnóstico y manejo tempranos (7).

Debido a la importancia de lesiones dermatológicas en pacientes críticos y la existencia de escasos reportes en la literatura sobre la incidencia $(9,12,15)$, se decide realizar el presente estudio, para describir la frecuencia de lesiones dermatológicas en pacientes de UCI en nuestro medio.

\section{Pacientes y métodos}

\section{Población de estudio y diseño del estudio}

Se efectuó un estudio descriptivo de corte transversal en las UCI del Hospital de San José y Hospital Infantil Universitario de San José durante cuatro meses (enero-abril de 2012).

Se valoraron pacientes mayores de 18 años que fueron interconsultados por aparición de lesiones en piel o ingresaron a la UCI por causa dermatológica. La estancia en la UCI debía ser como mínimo de 72 horas.

Las variables evaluadas están descritas en la Tabla 1, e incluyen: edad, sexo, motivo de ingreso, el tipo de lesión dermatológica (primaria, secundaria o terciaria, según clasificación propuesta por Dunnill) (7), comorbilidades, índice de masa corporal, entre otras.

La valoración fue realizada en turnos mensuales de cinco días por semana por dermatólogos de experiencia que realizaban la inspección del paciente, consignaban los datos en el formato de registro y en algunos casos fotografiaban la lesión. Se excluyeron pacientes quemados, o que no aceptaran participar (Tabla 1).

\section{Procesamiento de datos y análisis}

Los datos fueron recolectados en el instrumento diseñado para tal fin y tabulados para análisis estadístico en Microsoft Excel ${ }^{\circledR}$ versión 2007 . Las variables cualitativas se presentaron con frecuencias absolutas y relativas; las variables cuantitativas se resumieron con medidas de tendencia central y de dispersión. El análisis estadístico se realizó usando Stata ${ }^{\circledR} 12$.

\section{Aspectos éticos}

Este trabajo siguió las pautas internacionales y la normativa nacional vigentes en la materia $(22,23)$. Por tanto, toda la información recolectada incluyendo las fotografías se obtuvo bajo consentimiento informado y se manejó confidencialmente. El protocolo fue aprobado por el Comité de Investigaciones y Ética de la Facultad de Medicina de la Fundación Universitaria de Ciencias de la Salud.

\section{Resultados}

Durante el periodo comprendido entre enero y abril de 2012 se valoraron 595 pacientes hospitalizados en las UCI descritas, de los cuales 182 presentaron lesiones dermatológica (Tabla 1). No se encontraron diferencias en frecuencia por género, siendo el $50.2 \%$ de sexo femenino. La edad promedio fue 63.5 años \pm 19.1 (rango: 18-112). La mayoría ingresaron a la UCI por causas no quirúrgicas (57.3 vs. $42.6 \%$ ) (Tabla 2). De los 182 pacientes, 142 (77.6\%) 
Tabla 1. Características generales.

\begin{tabular}{|cc|}
\hline Lesiones dermatológicas & $182(30.5 \%)$ \\
\hline Edad promedio $^{*}$ & 63,5 \\
\hline Sexo (masculino) & $91(49,73 \%)$ \\
\hline Motivo de ingreso & $105(57,38 \%)$ \\
\hline No quirúrgico & $14(7.65 \%)$ \\
\hline IMC $<30 \mathrm{Kg} / \mathrm{m}^{2}$ & \\
\hline Comorbilidades & $38(26,23 \%)$ \\
\hline Diabetes mellitus & $3(1,64 \%)$ \\
\hline VIH & \\
\hline *Años & \\
\hline
\end{tabular}

tenían enfermedades de base (diabetes mellitus, hipertensión arterial, insuficiencia renal crónica, enfermedad pulmonar obstructiva crónica, enfermedad cerebrovascular, hipotiroidismo, insuficiencia cardiaca congestiva, hiperuricemia y trauma), encontrando un importante porcentaje de diabetes asociado a las manifestaciones cutáneas (Tabla 2).

\section{Lesiones primarias}

De los 182 pacientes, sólo tres (1.6\%) presentaron lesiones primarias que fueron suficiente criterio para el ingreso a UCI: SSJ $(n=1)$, eritema multiforme $(n=1)$ y pénfigo vulgar $(\mathrm{n}=1)$ (Figuras 1 y 2$)$.

\section{Lesiones secundarias}

De los pacientes evaluados, once $(6 \%)$ cursaban con lesiones secundarias, encontrándose una o más de las siguientes: púrpura trombocitopénica, púrpura fulminante, herpes zoster, púrpura senil, úlcera varicosa, celulitis por micobacterias, foliculitis bacteriana, tiña corporis y celulitis bacteriana.

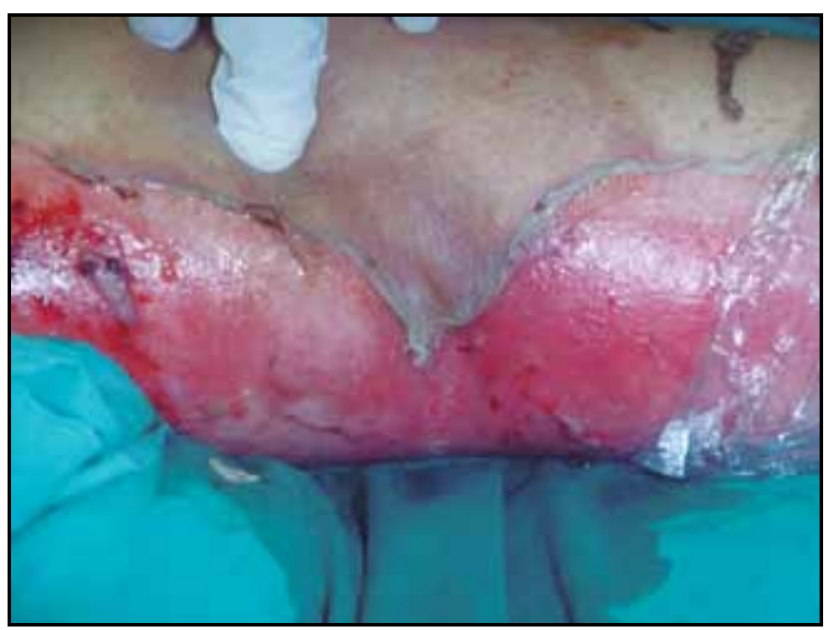

Figura 1. Pénfigo Vulgar. Paciente con extensa erosión en tronco, posterior a ruptura de ampollas.

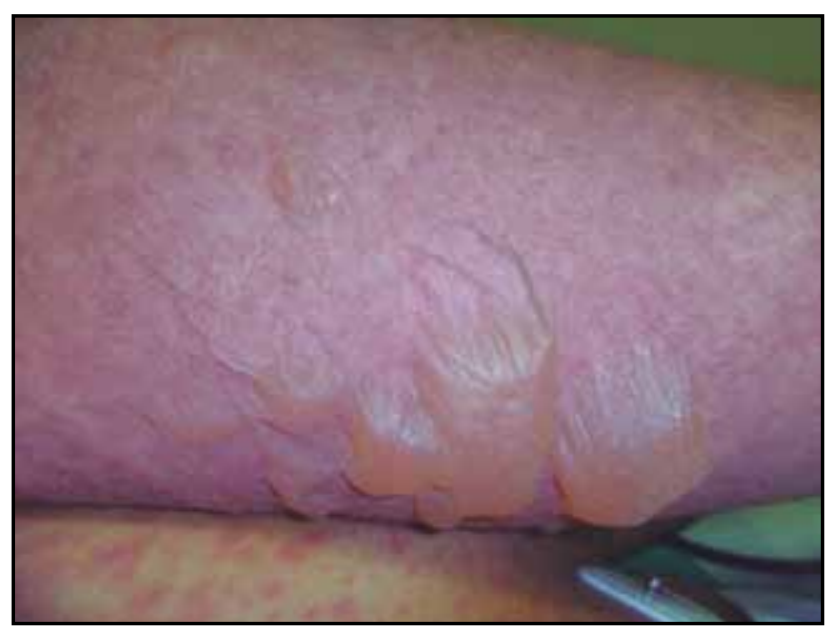

Figura 2. Síndrome de Stevens Johnson. Ampollas flácidas sobre una base eritematosa y edematosa comprometiendo el antebrazo. Nótese además las máculas eritematosas y violáceas afectando el pecho.

Tabla 2. Características demográficas de acuerdo con el tipo de lesión dermatológica.

\begin{tabular}{|c|c|c|c|c|c|c|}
\hline & $\begin{array}{c}\text { Lesión } \\
\text { primaria } \\
(3 \text { pacientes })\end{array}$ & $\begin{array}{c}\text { Lesión } \\
\text { secundaria } \\
\text { (11 pacientes) }\end{array}$ & $\begin{array}{c}\text { Lesión } \\
\text { terciaria } \\
\text { (168 pacientes) }\end{array}$ & $\begin{array}{c}\text { E. } \mathbf{V}^{g} \\
\text { (1 paciente) }\end{array}$ & $\begin{array}{c}\text { E.B }{ }^{* *} \\
\text { (7 pacientes) }\end{array}$ & $\begin{array}{c}\text { E.M }^{\dagger \dagger} \\
\text { (9 pacientes) }\end{array}$ \\
\hline Edad (años) & $40 \pm 20$ & $63 \pm 25$ & $62 \pm 18$ & $78 \pm 0$ & $53 \pm 21$ & $58 \pm 12$ \\
\hline \multicolumn{7}{|l|}{ Sexo } \\
\hline *Masculino & $1 / 3$ & $9 / 11$ & $81 / 168$ & $1 / 1$ & $4 / 7$ & $4 / 9$ \\
\hline \multicolumn{7}{|l|}{ Motivo de ingreso } \\
\hline *No quirúrgico & $3(100 \%)$ & $8(72 \%)$ & $94(56 \%)$ & 0 & $6(85 \%)$ & $6(66 \%)$ \\
\hline \multicolumn{7}{|l|}{ Comorbilidades } \\
\hline *Diabetes mellitus & $1(33 \%)$ & $4(42 \%)$ & $43(25 \%)$ & 0 & $3(42 \%)$ & $1(11 \%)$ \\
\hline$* \mathrm{VIH}$ & 0 & 0 & $3(1 \%)$ & 0 & 0 & 0 \\
\hline \multicolumn{7}{|c|}{$\begin{array}{l}\text { † Lesiones Primarias: Entidades dermatológicas intrínsecas que conducen a la hospitalización. } \\
\text { † Lesiones secundarias: Manifestaciones en la piel de enfermedades sistémicas que conducen a la hospitalización } \\
\text { \&esiones terciarias: Manifestaciones en la piel producto de los procedimientos durante la hospitalización. } \\
\text { I: Enfermedades virales; **: Enfermedades por bacterias; } † \text { † Enfermedades micóticas }\end{array}$} \\
\hline
\end{tabular}


De los que cursaron con enfermedad bacteriana, uno tuvo compromiso por estreptococo $(0.5 \%)$ y cinco $(2.7 \%)$ por infección mixta (dos o más agentes microbianos). Se encontraron siete (3.8\%) con infección por Candida spp; no se encontraron enfermedades parasitarias de la piel en toda la muestra.

\section{Lesiones terciarias}

De 168 pacientes $(91.8 \%)$ que presentaron lesiones terciarias, se encontró que 25 (13.6\%) tenían úlceras o zonas de presión en región sacra, codos, maléolos o talones; 41 pacientes $(22.4 \%)$, presentaron dermatitis de contacto secundaria a pegamento de electrodos. No se encontró urticaria ni vasculitis en ninguno de los pacientes evaluados (Figuras 3 y 4 ).

Cinco pacientes $(2.9 \%)$ recibieron manejo con medicamentos inmunosupresores: cuatro $(2.1 \%)$ recibieron corticoides y uno $(0.5 \%)$ azatioprina.

Tres pacientes (1.6\%) cursaron con infección por VIH en tratamiento con antirretrovirales; dos $(1.0 \%)$ presentaron enfermedades autoinmunes y ninguno tenía antecedentes de tratamiento previo con medicamentos biológicos.

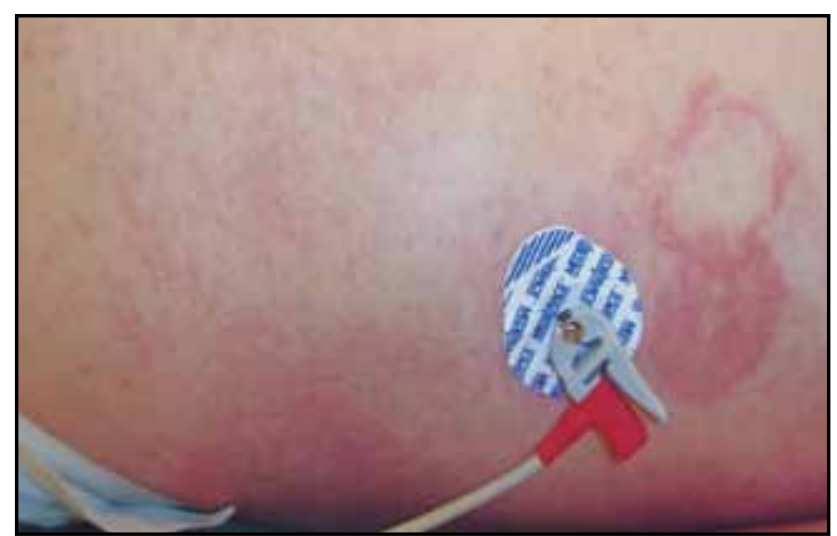

Figura 3. Úlcera por presión. Úlcera superficial bien delimitada que compromete la región sacra.

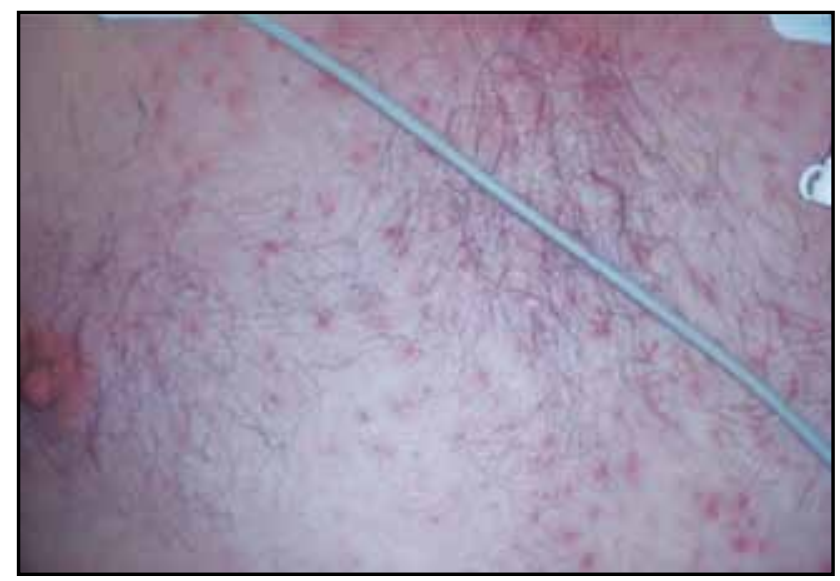

Figura 5. Foliculitis. Múltiples pápulas eritematosas foliculares y escasas pústulas afectando el tórax anterior.
Del total de pacientes evaluados, $23(12.5 \%)$ murieron, dos de éstos por causa dermatológica (un caso de pénfigo vulgar y otro de SSJ); 160 pacientes $(87.4 \%)$ continuaron manejo intrahospitalario en la UCI o fueron trasladados a otros servicios después de la valoración (Figura 5 y 6).

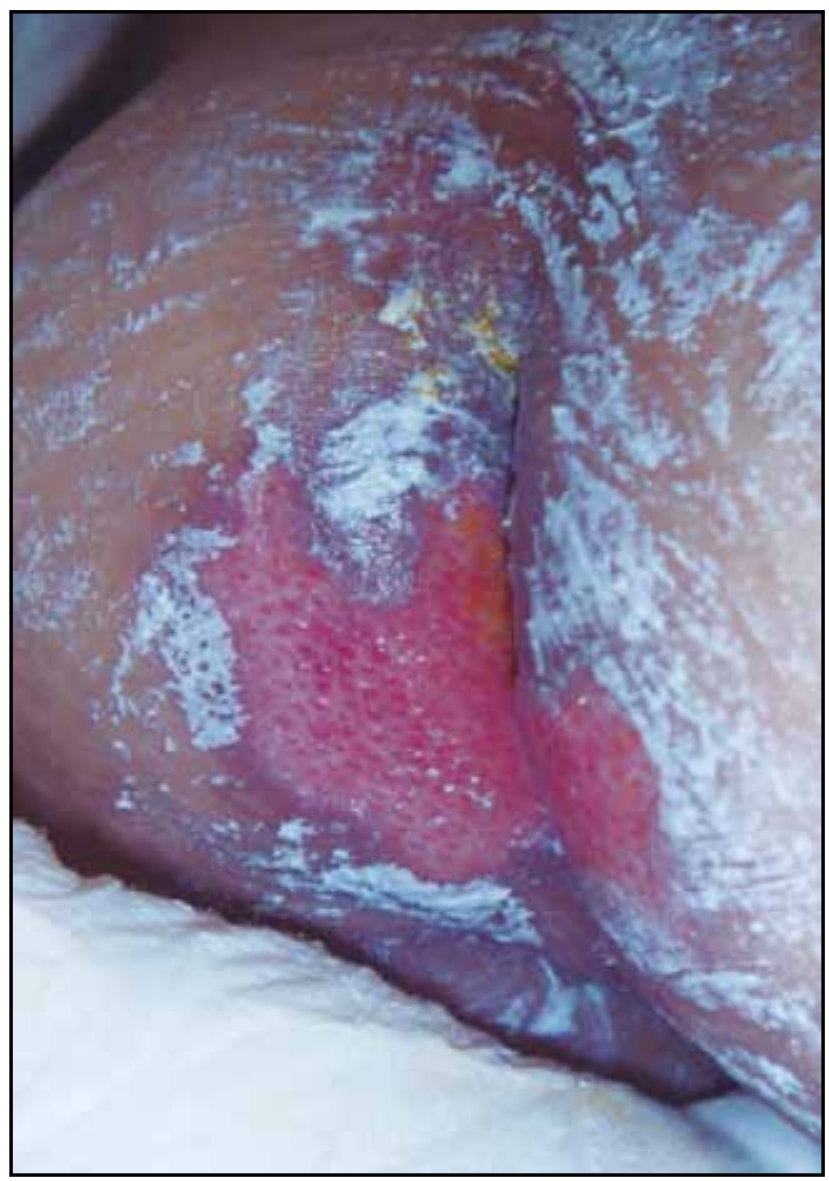

Figura 4. Dermatitis de contacto a pegamento de electrodos. Placas anular y numular eritematosas coalescentes, bien delimitadas que comprometen región torácica y aparecen en sitio de contacto a electrodos.

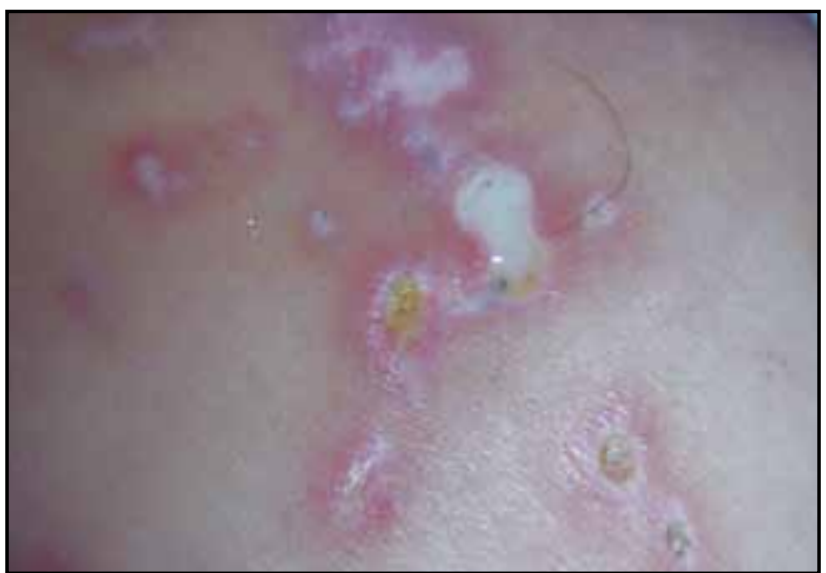

Figura 6. Sobreinfección del sitio de inserción de catéter central. Se evidencian las pequeñas úlceras de bordes eritematosos que confluyen hacia el sitio de inserción de catéter subclavio, algunas se encuentran cubiertas por costra amarillenta y otras presentan secreción purulenta. 


\section{Discusión}

Las lesiones dermatológicas se encuentran hasta en 30\% de los pacientes de UCI y debido al alto riesgo de inducción de falla cutánea, ameritan un diagnóstico y tratamiento temprano, así como un manejo multidisciplinario que evite resultados fatales.

El origen de las lesiones es multifactorial, por ejemplo, reacciones cutáneas adversas a medicamentos las cuales son benignas y transitorias; sin embargo, pocos casos son graves y potencialmente mortales, requiriendo la mayoría soporte en UCI $(24,25)$.

En el presente estudio se encontraron diversas patologías cutáneas, tales como: celulitis, foliculitis causada por estreptococo, infección bacteriana mixta o infecciones micóticas por Candida spp. La importancia de dichos hallazgos estriba en que los primeros signos de sepsis pueden aparecer en la piel, pero su especificidad es escasa, así que es posible encontrar lesiones iniciales como máculas eritematosas, ampollas, nódulos o inclusive nódulos necróticos, las cuales pueden ser motivo de alerta para el clínico y tienen que ser vigiladas cuidadosamente $(7,26)$.

Los pacientes hospitalizados en la UCI pueden ser más susceptibles a sufrir algún tipo de infección cutánea, a pesar de lo inespecífico de los síntomas, signos o microorganismos causantes $(15,27-29)$, estos resultados fueron similares a los reportados por Atuesta y Campos-Fernández (9).

Muchos de los pacientes en estado crítico, reciben varios medicamentos durante su hospitalización, aumentando así el riesgo de reacciones por drogas, lo que hace importante su identificación precoz para la suspensión o reemplazo $(7,10,12,30)$, en este estudio se encontraron reacciones medicamentosas como eritema multiforme, secundario a uso de fenitoína.

Las lesiones más frecuentes en este estudio fueron las dermatitis de contacto, úlceras y zonas de presión, con incidencias que varían entre 4.7 y $18.6 \%$. Estos hallazgos coinciden con los observados por Badia et al (4) donde las primeras se dan por el contacto a elementos de fijación y cobertura (cinta adhesiva, pegamento de los electrodos).

Además encontramos que un porcentaje importante de los pacientes hospitalizados en la UCI presentaban algún tipo de lesión dermatológica, ocasionada por diversas situaciones clínicas, asociadas o no a enfermedades sistémicas, o concomitantes con éstas tal como se ha referido en la literatura $(7,16)$. Encontramos dos casos con lesiones primarias cuyo resultado fue fatal y con mucha frecuencia encontramos zonas de presión y úlceras.

Consideramos que aunque en las unidades donde se realizó el estudio existen protocolos rigurosos de movilidad periódica, mínimo cada dos horas, estos pacientes presentaron muchos factores de riesgo asociados que predisponen su aparición como: desnutrición, disminución local del aporte sanguíneo, choque séptico, historia de enfermedad oclusiva arterial periférica y pérdida de la sensibilidad (7, 31-33).
Es claro que la falta de movilidad constituye la principal causa desencadenante para el desarrollo de zonas y úlceras de presión, por lo tanto, su control es un indicador de calidad en la gestión de procesos de enfermería en ambas unidades, sin embargo, no podemos afirmar si las lesiones de este tipo encontradas en nuestro estudio fueron desarrolladas durante la estancia en UCI o si los pacientes ya las presentaban en el momento del ingreso. Las limitantes identificadas en el estudio, fueron el difícil acceso a toda la superficie cutánea del paciente, así como la falta de disponibilidad permanente del dermatólogo evaluador en la UCI, lo que impidió establecer claramente una relación temporal con la aparición de las lesiones. Teniendo en cuenta estos resultados consideramos fundamental desarrollar estrategias y guías de control terapéutico para estos casos, las cuales sean realizadas conjuntamente entre los servicios de dermatología y UCI, y evaluar su adherencia comparada con el éxito o fracaso del manejo.

En conclusión, es pertinente hacer un seguimiento dermatológico en los pacientes hospitalizados en estas unidades para identificar y tipificar las afecciones dermatológicas en UCI.

\section{Agradecimientos}

Los autores del artículo quieren agradecer a los doctores Ingrid Ángulo, Luisa Castaño, Viviana Garzón, Sebastián Vélez, Mónica Novoa Candía y Miguel Olmos Pérez, por sus aportes para la construcción de este documento.

\section{Referencias}

1. Samudrala S, Dandakeri S, Bhat RM. Clinical profile of dermatological emergencies and intensive care unit admissions in a tertiary care center - an Indian perspective. Int J Dermatol. 2018;57(5):575-9.

2. Alani A, Sadlier M, Uddin A, Hackett C, Ramsay B, Ahmad K. An analysis of inpatient dermatologic consultations at University Hospital Limerick: inadequate infrastructure leads to acute skin failure. Ir J Med Sci. 2017;186(2):305-7.

3. Hassan I, Rather PA. Emergency dermatology and need of dermatological intensive care unit (DICU). Journal of Pakistan Association of Dermatology. 2013;23(1):71-82

4. Badia M, Servia L, Casanova JM, Montserrat N, Vilanova J, Vicario E, et al Classification of dermatological disorders in critical care patients: a prospective observational study. Journal of critical care. 2013;28(2):220 e1-8.

5. Inamadar AC, Palit A. Acute skin failure: concept, causes, consequences and care. Indian journal of dermatology, venereology and leprology. 2005;71(6):379-85.

6. Thomas M, Peter JV, Williams A, Job V, George R. Systemic inflammatory response syndrome in diseases of the skin. Postgraduate medical journal. 2010;86(1012):83-8.

7. Dunnill MG, Handfield-Jones SE, Treacher D, McGibbon DH. Dermatology in the intensive care unit. The British journal of dermatology. 1995;132(2):226-35.

8. Rosenbach M. Skin Rashes. The Intensive Care Unit Manual. Second ed: Elsevier; 2014. p. 424-33.

9. Atuesta Negret J. Semiología del sistema tegumentario en el paciente crítico Acta Colombiana de Cuidado Intensivo. 2009;10(2):101-10.

10. Cañarte C, Valencia N, Collantes J. Manifestaciones dermatológicas de los trastornos sistémicos. In: FEPSO Fedlp, editor.

11. Janniger CK, Schwartz RA, Szepietowski JC, Reich A. Intertrigo and common secondary skin infections. American family physician. 2005;72(5):833-8.

12. Chang P, Sánchez Y, Rodríguez R. Dermatosis observadas en una Unidad de Terapia Intensiva. Dermatología Cosmética, Clínica y Quirúrgica; 2014.

13. Lin LH, Hopf HW. Paradigm of the injury-repair continuum during critical illness. Critical care medicine. 2003;31(8 Suppl):S493-5.

14. Williams DT, Harding K. Healing responses of skin and muscle in critical illness. Critical care medicine. 2003;31(8 Suppl):S547-57.

15. Peña Á, Peñaranda E, Sánchez A. Enfermedades Dermatológicas de Pacientes Hospitalizados en Cuidados Intensivos Hospital de la Samaritana. 2016. 
16. Badia M, Trujillano J, Gasco E, Casanova JM, Alvarez M, Leon M. Skin lesions in the ICU. Intensive Care Medicine. 1999;25(11):1271-6.

17. George SM, Harrison DA, Welch CA, Nolan KM, Friedmann PS. Dermatological conditions in intensive care: a secondary analysis of the Intensive Care National Audit and Research Centre (ICNARC) Case Mix Programme database. Crit Care. 2008;12 Suppl 1:S1.

18. Irwin RS, Rippe JM, Lilly CM. Irwin \& Rippe's Manual of Intensive Care Medicine. Sixth edition ed. Philadelphia: Wolters Kluwer; 2014.

19. Wanat KA, Kovarik CL, Fett N. Pustular eruption in the ICU. The American Journal of Medicine. 2012;125(8):758-60.

20. Kanji S, Chant C. Allergic and hypersensitivity reactions in the intensive care unit. Critical Care Medicine 2010; 38(6 Suppl): S162-8.

21. Schnyder B. Approach to the patient with drug allergy. Immunology and allergy clinics of North America. 2009;29(3):405-18.

22. Asamblea W, Mundial M. Declaración de Helsinki: Investigación médica en seres humanos. 2013

23. de R, Colombia MdS. Resolución 008430 de 1993. Por la cual se establecen las normas científicas, técnicas y administrativas para la investigación en salud. 1993.

24. Kumar R, Das A, Das S. Management of Stevens-Johnson SyndromeToxic Epidermal Necrolysis: Looking Beyond Guidelines! Indian J Dermatol. 2018;63(2):117-24

25. Harr T, French LE. Toxic epidermal necrolysis and Stevens-Johnson syndrome. Orphanet J Rare Dis 2010;5:39.

26. Leon C, Ruiz-Santana S, Saavedra P, Galvan B, Blanco A, Castro C, et al.
Usefulness of the "Candida score" for discriminating between Candida colonization and invasive candidiasis in non-neutropenic critically ill patients: a prospective multicenter study. Critical Care Medicine. 2009;37(5):1624-33.

27. Campos-Fernandez Mdel M, Ponce-De-Leon-Rosales S, Archer-Dubon C, Orozco-Topete R. Incidence and risk factors for cutaneous adverse drug reactions in an intensive care unit. Revista de investigación clínica; órgano del Hospital de Enfermedades de la Nutrición. 2005;57(6):770-4.

28. Wolf R, Orion E, Marcos B, Matz H. Life-threatening acute adverse cutaneous drug reactions. Clin Dermatol. 2005;23(2):171-81.

29. Vardakas KZ, Samonis G, Michalopoulos A, Soteriades ES, Falagas ME. Antifungal prophylaxis with azoles in high-risk, surgical intensive care unit patients: a meta-analysis of randomized, placebo-controlled trials. Crit Care Med. 2006;34(4):1216-24.

30. Jack AR, Spence AA, Nichols BJ, Peng DH. A simple algorithm for evaluating dermatologic disease in critically ill patients: a study based on retrospective review of medical intensive care unit consults. J Am Acad Dermatol. 2009;61(4):728-30.

31. Badia M, Trujillano J, Gascó E, Casanova JM, Alvarez M, León M. Skin lesions in the ICU. Intensive Care Med. 1999;25(11):1271-6.

32. Dünser MW, Mayr AJ, Tür A, Pajk W, Barbara F, Knotzer H, et al. Ischemic skin lesions as a complication of continuous vasopressin infusion in catecholamine-resistant vasodilatory shock: incidence and risk factors. Crit Care Med. 2003;31(5):1394-8.

33. Fischer M, Soukup J, Wohlrab J, Radke J, Marsch W. Key dermatological symptoms in the intensive care unit. Int J Dermatol. 2004;43(10):780-2. 